\title{
Comparación del análisis de orina por el método manual y el automatizado
}

\author{
Comparison of urine analysis by manual and automated method
}

María Alexandra Montalvo Torres. ${ }^{1}$, Melissa Andrea Peralta Mosquera. ${ }^{2}$, Santiago José Robalino Montalvo. ${ }^{3} \&$ Marisol Bertha Ordoñez Revelo. ${ }^{4}$

Recibido: 31-05-2019 / Revisado: 24-06-2019 /Aceptado: 01-08-2019/ Publicado: 06-09-2019

\begin{abstract}
DOI: _ https://doi.org/10.33262/cienciadigital.v3i3.3.791

Urinalysis is frequently requested in clinical laboratories. It allows to determine the existence of kidney problems, disorders of the urinary system, as well as the general functioning of the organism by determining the metabolites of the urine and the presence of bacteria, cells, among others in the sediment. It is a visual, chemical and microscopic examination that demands the necessary expertise of the analyst. Its manual execution involves time, is laborious and is linked to numerous errors. For this reason, automated systems become the best option. Among the advantages are: standardization of methods, decrease in variation associated with the observer, obtaining immediate results, increase in the number of samples processed and elimination of errors by transcription. An observational study was conducted to obtain information on concordance and accuracy. of the AutionHybrid AU-4050 automated equipment in 100 samples that met the inclusion and exclusion criteria at the Pablo Arturo Suárez Hospital. It was concluded that this automated urinalysis system has excellent performance. It is suggested the implementation of this sanitary technology in laboratories for all the benefits that were evidenced. Thus, patients and professionals will benefit as decisions will be expedited in therapeutic behaviors. In addition, work in the laboratory will be optimized.
\end{abstract}

Keywords: Urinalysis, automated systems, sediment, microscopy, precision, concordance

\section{Resumen}

El urianálisis se solicita frecuentemente en los laboratorios clínicos. Permite determinar la existencia de problemas renales, trastornos del sistema urinario, así como el funcionamiento general del organismo mediante la determinación de los metabolitos de la orina y la presencia de bacterias, células, entre otros en el sedimento. Es un examen visual, químico y microscópico que demanda de una necesaria experticia del analista. Su ejecución manual implica tiempo, es laboriosa y está ligada

\footnotetext{
${ }^{1}$ Hospital Pablo Arturo Suárez, Quito Ecuador, draalexmontalvo@ hotmail.com

${ }^{2}$ SIMED, Quito Ecuador, meli_759@hotmail.com

${ }^{3}$ Universidad de las Américas, Quito-Ecuador, robalinosanti@outlook.com

4 Hospital Pablo Arturo Suárez, Quito Ecuador, holasol68@yahoo.com
} 
a numerosos errores. Por este motivo, los sistemas automatizados se convierten en la mejor opción. Entre las ventajas están: estandarización de los métodos, disminución de la variación asociada al observador, obtención de resultados inmediatos, incremento en el número de muestras procesadas y eliminación de errores por transcripción. Se realizó un estudio observacional para obtener información sobre la concordancia y precisión del equipo automatizado AutionHybrid AU-4050 en 100 muestras que cumplieron los criterios de inclusión y exclusión en el Hospital Pablo Arturo Suárez. Se concluyó que este sistema automatizado de urianálisis tiene un excelente desempeño. Se sugiere la implementación de la esta tecnología sanitaria en los laboratorios por todos los beneficios que se evidenciaron. Así, se beneficiará a los pacientes y a los profesionales pues se agilitará la toma de decisiones en las conductas terapéuticas. Además, se optimizará el trabajo en el laboratorio.

Palabras clave: Urianálisis, sistemas automatizados, sedimento, microscopía, precisión, concordancia

\section{Introducción}

El examen de orina es un análisis que se solicita frecuentemente en los laboratorios clínicos debido a la importancia en el diagnóstico clínico. El urianálisis permite determinar la existencia de problemas renales, trastornos del sistema urinario así como el funcionamiento general del organismo mediante la determinación del desbalance en los metabolitos de la orina y la presencia de bacterias, células entre otros en el sedimento.(1)

Es un examen visual, químico y microscópico que demanda de una necesaria experticia del analista. La lectura manual de las tirillas y el análisis del sedimento urinario resultan laboriosos y tienen poca precisión debido a las variaciones de la preparación y a la técnica del contaje. Sumado a estos elementos debemos considerarse que la falta de una metodología de control de calidad apropiada y número elevado de muestras podría generar errores en las etapas de análisis pre - analítica, analítica y/o post - analítica.(2)

Por este motivo y considerando la importancia de asegurar la calidad en la atención acompañada de un alto estándar de eficiencia en los procesos analíticos, actualmente el uso de sistemas automatizados de orinas tiene que convertirse en la mejor opción para cumplir estos objetivos.(3)

Otras ventajas de la automatización son la rapidez en la respuesta, incremento en el número de pruebas que realiza el laboratorio clínico esto último producto de una mejor planificación y un control de calidad automatizado.(3)(4)

Los sistemas automatizados de orinas además permiten que el trabajo microscópico sea destinado al análisis de orinas patológicas y evita la transcripción de resultados. De esta forma el analista tiene el tiempo necesario para la realización de los mantenimientos programados del equipo, estadísticas y otras actividades inherentes al trabajo del laboratorio clínico.(4) La implementación de sistemas automatizados optimiza el tiempo y los recursos en los laboratorios de alta demanda.(5)

El presente estudio tiene los siguientes objetivos: 
- Realizar una validación y verificación del funcionamiento del equipo automatizado del laboratorio.

- Emplear estudios de precisión y concordancia siguiendo los protocolos definidos por el fabricante, con el fin de comprobar su utilidad en las actividades rutinarias del laboratorio.

\section{Materiales y métodos}

Se realizó un estudio observacional en el laboratorio clínico del Hospital Pablo Arturo Suárez.

\section{Muestra:}

Por tratarse de un universo infinito y homogéneo, se utilizó el muestreo aleatorio simple para variable cualitativa. Se recolectaron 100 muestras de pacientes ambulatorios y hospitalizados a quienes se les solicitó el examen elemental y microscópico de orina. No se consideró la sintomatología.

\section{Criterios de inclusión:}

Las muestras deben ser recogidas en un frasco estéril de boca ancha; realizar higiene previa a la obtención de la orina; evitar estar en periodo menstrual, recolectar la primera muestra de orina de la mañana y que corresponda al chorro medio de la micción, transportar al laboratorio en un máximo de dos horas o conservar en refrigeración de 2 a 8 grados hasta por 24 horas.(6)

\section{Criterios de exclusión}

Muestras recogidas en envases no adecuados, problemas en identificación de las muestras, que tengan más de dos horas de recolectadas y que no hayan estado en refrigeración y que se encuentren contaminadas con heces.

Procedimiento para el estudio de precisión: Se investigó la reproducibilidad de los resultados que reporta el analizador, tanto en la sección CHM (sistema automatizado que realiza análisis de tiras de ensayo) como en la sección FCM (sistema automatizado que realiza análisis de partículas). Se emplearon los siguientes materiales: analizador AU-4050, dos niveles de control de calidad para química CHM, dos niveles de control de calidad para sedimento FCM y hojas de cálculo para determinar la precisión (diseñadas por el fabricante). Se realizó la siguiente metodología:

1. Se verificó que todo el mantenimiento y calibración aplicable se haya realizado en el instrumento.

2. Se utilizó el equipo de protección personal indicado (bata de laboratorio, guantes y gafas de seguridad).

3. Se llevó los controles de calidad fuera del refrigerador y permitir que alcancen la temperatura ambiente.

4. Se llenó los tubos de ensayo con volumen adecuado de control de la calidad y ubicar en un rack de muestras.

5. Se configuró el instrumento para informar resultados en reflectancia.

6. Se completó un total de 21 réplicas, tanto de los controles bajo y alto de química. 
7. Se completó un total de 21 réplicas, tanto de control bajo y alto de sedimento.

8. Se completó la hoja de cálculo de precisión con los valores obtenidos de los controles de química.

9. Se completó la hoja de cálculo de precisión con los valores obtenidos de los controles FCM.

Las hojas de cálculo de precisión calcularon automáticamente la media, desviación estándar y el coeficiente de variación. Se analizó si los resultados obtenidos estaban en los rangos asignados por el fabricante.

Procedimiento para el estudio de concordancia: Permite determinar la exactitud de los resultados reportados por el analizador en ambas secciones CHM y FCM. Se emplearon los siguientes materiales: analizador AU-4050, tiras de ensayo manuales, microscopio y hojas de datos electrónicas para CHM y FCM, éstas últimas diseñadas por el fabricante. Se realizó la siguiente metodología:

1. Se procesaron las muestras en el analizador y posteriormente se realizó la lectura manual utilizando tiras auxiliares para química y la microscopía para el sedimento.

2. Ingrese los resultados en las hojas de datos electrónicas para CHM y FCM respectivamente. Compare los resultados de cada analito y determine si se supera el criterio de aceptación ( $85 \% \pm 1$ bloque de color).

\section{Resultados}

El estudio de precisión demostró que el analizador AutionHybrid AU-4050 tiene un comportamiento aceptable en cuanto a repetibilidad de resultados, tanto en CHM como en FCM. Los CV no superaron los límites declarados por el fabricante en la mayoría de los analitos con excepción del urobilinógeno. La precisión media de este último fue del 65,04\%. Está establecido que el valor del CV debe ser menor que el que corresponde a la aceptación (ver tablas 1 y 2 ).

Tabla 1. Resultado de la precisión del análisis químico urinario (CHM) Nivel 1

\begin{tabular}{|c|c|c|c|c|c|c|c|c|c|c|}
\hline & GLU & PRO & BIL & URO & $\mathrm{pH}$ & BLD & KET & NIT & LEU & $\begin{array}{l}\text { Velocidad de } \\
\text { sedimentación }\end{array}$ \\
\hline Media & 92,77 & 75,09 & 96,07 & 65,04 & 25,7 & 89,91 & 50,98 & 99,47 & 62,1 & 1015 \\
\hline $\begin{array}{l}\text { Desviación } \\
\text { estándar }\end{array}$ & 0,49 & 0,9 & 0,45 & 5,44 & 0,56 & 0,83 & 1,22 & 0,22 & 2,74 & 0,707 \\
\hline $\begin{array}{l}\text { Porcentaje } \\
\text { de CV* }\end{array}$ & 0,53 & 1,2 & 0,47 & 8,37 & 2,17 & 0,93 & 2,39 & 0,23 & 4,41 & 0,07 \\
\hline Aceptación & 0,78 & 1,62 & 0,51 & 5,38 & 2,71 & 3,63 & 2,45 & 0,59 & 5,1 & 0,07 \\
\hline Estado & APRUEBA & APRUEBA & APRUEBA & $\begin{array}{c}\text { NO } \\
\text { APRUEBA }\end{array}$ & APRUEBA & APRUEBA & APRUEBA & APRUEBA & APRUEBA & APRUEBA \\
\hline
\end{tabular}

Fuente: Informe de validación del equipo AUTION HYBRID AU-4050, Hospital Pablo Arturo Suárez, 2015

*Coeficiente de variación 
Tabla 2. Resultado de precisión del análisis de partículas de orina (FCM) Nivel 1

\begin{tabular}{|c|c|c|c|c|c|}
\hline & RBC & WBC & EC & CAST & $\begin{array}{l}\text { BACT } \\
(6 \mathrm{uL} \text { modo) }\end{array}$ \\
\hline Media & 34,92 & 40,78 & 10,69 & 4,79 & 184,21 \\
\hline Desviación estándar & 3,21 & 2,69 & 1,90 & 0,95 & 13,39 \\
\hline Porcentaje de $\mathrm{CV}^{*}$ & 9,18 & 6,59 & 17,78 & 19,78 & 7,27 \\
\hline Aceptación & 10,0 & 10,0 & 30,0 & 40,0 & 10,0 \\
\hline Estado & APRUEBA & APRUEBA & APRUEBA & APRUEBA & APRUEBA \\
\hline
\end{tabular}

Fuente: Informe de validación del equipo AUTION HYBRID AU-4050, Hospital Pablo Arturo Suárez, 2015

*Coeficiente de variación

Se compararon los resultados obtenidos por el equipo y los interpretados por el operador en la tirilla y en la microscopía manual. En el gráfico 1, se presenta la correlación lineal de la densidad urinaria. Los puntos están próximos a la línea recta. En aquellos más alejados, se establece que no hay concordancia. En casi todos los otros parámetros la concordancia superó el $80 \%$ que se considera como criterio de aceptación. En la tabla 3 se muestra los resultados de precisión del equipo para glucosa. La concordancia fue del $92 \%$, es decir que superó el $80 \%$. En la tabla 4 se indica que en el caso de las bacterias la concordancia $66 \%$ porque el equipo reportó 17 muestras negativas mientras en el microscopio se reportaron con $1+$.

Figura 1. Correlación lineal de la densidad

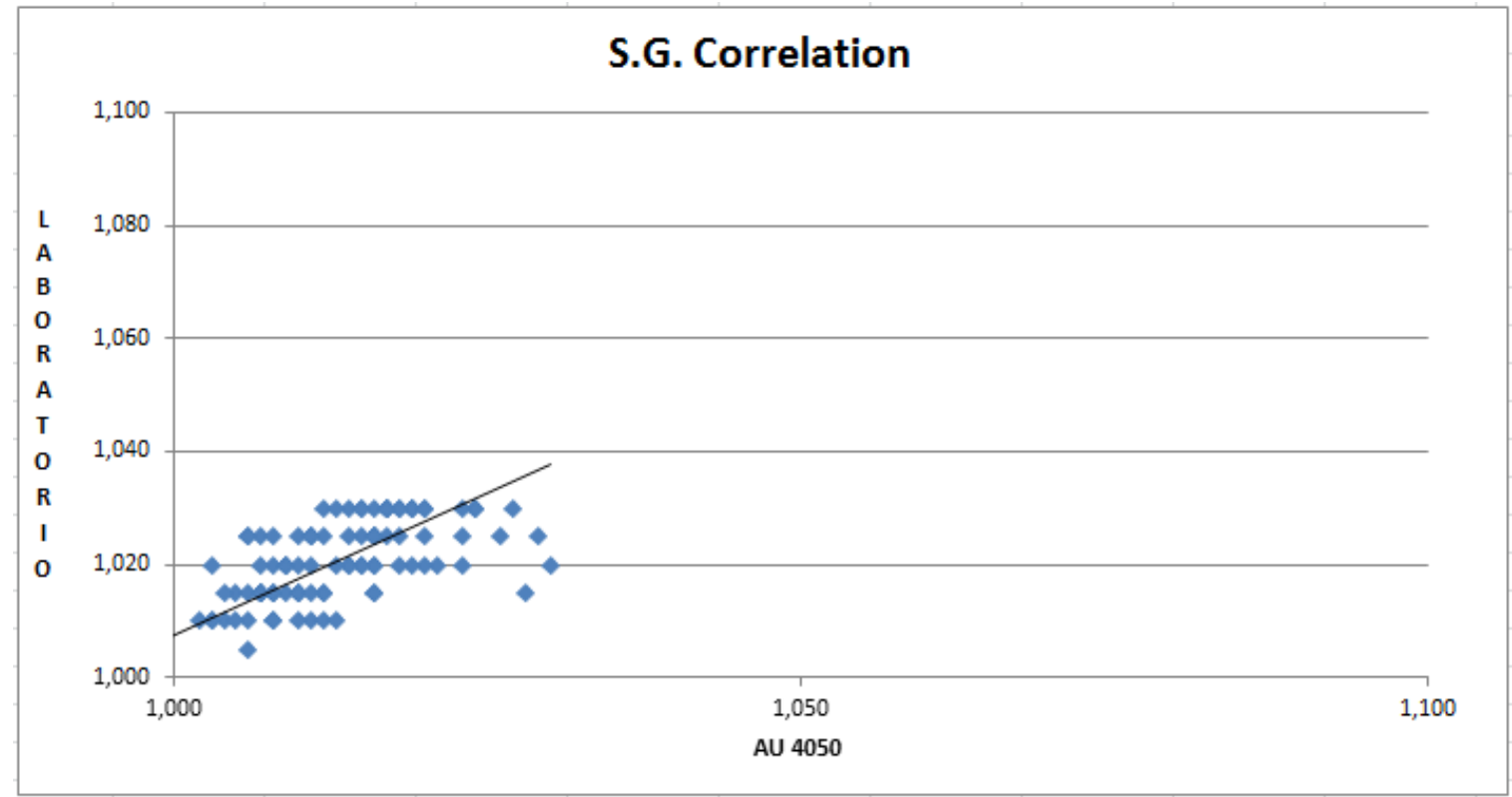

Fuente: Informe de validación del equipo AUTION HYBRID AU-4050, Hospital Pablo Arturo Suárez, 2015. 
Tabla 3. Análisis de concordancia del equipo para la glucosa.

\section{GLUCOSA (mg/dL)}

REFERENCIA: LABORATORIO

\begin{tabular}{|c|c|c|c|c|c|c|c|}
\hline \multirow{7}{*}{ 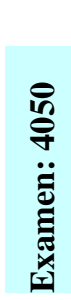 } & \multirow{3}{*}{ Negativo } & Negativo & ( \pm ) $30-50$ & (+) 70-100 & $(++) \mathbf{1 5 0 - 2 0 0}$ & $(+++) 300-500$ & $(++++) \geq 1000$ \\
\hline & & 91 & 1 & 0 & 0 & 0 & 0 \\
\hline & & 1 & 0 & 0 & 0 & 0 & 0 \\
\hline & (+) 70-100 & 1 & 1 & 0 & 0 & 0 & 0 \\
\hline & $(++) 150-200$ & 0 & 0 & 0 & 0 & 0 & 0 \\
\hline & $(+++) 300-500$ & 1 & 0 & 0 & 0 & 0 & 1 \\
\hline & $(++++) \geq 1000$ & 0 & 0 & 1 & 1 & 0 & 1 \\
\hline TO' & DTAL & 94 & 2 & 1 & 1 & 0 & 2 \\
\hline \multirow{2}{*}{\multicolumn{2}{|c|}{$\begin{array}{l}\text { Exactitud } \\
\text { Exactitud +/-1 }\end{array}$}} & $97 \%$ & 0\% & $0 \%$ & 0\% & $0 \%$ & $\mathbf{5 0 \%}$ \\
\hline & & $97 \%$ & $50 \%$ & $0 \%$ & $0 \%$ & $0 \%$ & $100 \%$ \\
\hline
\end{tabular}

Fuente: Informe de validación del equipo AUTION HYBRID AU-4050, Hospital Pablo Arturo Suárez, 2015

Tabla 4. Análisis de concordancia del equipo para bacterias

BACTERIAS

REFERENCIA: LABORATORIO

\begin{tabular}{|c|c|c|c|c|c|c|}
\hline \multirow{6}{*}{ 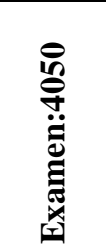 } & \multirow{3}{*}{$\begin{array}{l}\text { Negativo } \\
\pm\end{array}$} & \multirow{2}{*}{$\begin{array}{l}\text { Negativo } \\
54\end{array}$} & \multirow{2}{*}{$\begin{array}{l} \pm \\
0\end{array}$} & \multirow{2}{*}{$\begin{array}{l}\mathbf{1 +} \\
17\end{array}$} & \multirow{2}{*}{$\begin{array}{l}2+ \\
4\end{array}$} & \multirow{2}{*}{$\begin{array}{l}3+ \\
2\end{array}$} \\
\hline & & & & & & \\
\hline & & 2 & 0 & 2 & 2 & 1 \\
\hline & $1+$ & 0 & 0 & 1 & 0 & 1 \\
\hline & $2+$ & 1 & 0 & 0 & 3 & 0 \\
\hline & $3+$ & 0 & 0 & 0 & 2 & 8 \\
\hline TOTA & & 57 & 0 & 20 & 11 & 12 \\
\hline Exactit & & $95 \%$ & $0 \%$ & $5 \%$ & $27 \%$ & $67 \%$ \\
\hline Exactit & ud $+/-1 \mathrm{CB}$ & $95 \%$ & $0 \%$ & $15 \%$ & $45 \%$ & $67 \%$ \\
\hline
\end{tabular}

Fuente: Informe de validación del equipo AUTION HYBRID AU-4050, Hospital Pablo Arturo Suárez, 2015

\section{Discusión}

$\mathrm{Al}$ analizar la correlación de los resultados obtenidos de la densidad urinaria entre la lectura manual y automatizada, se debe considerar que los puntos más alejados podrían deberse a una posible subjetividad en la interpretación de los colores obtenidos en la tirilla por parte del observador.

En el caso de los niveles de urobilinógeno que no se encontraron en los límites de aceptación establecidos, debe considerarse que las siete primeras muestras se analizaron con un control que tenía algún tiempo de almacenamiento, mientras que las demás fueron procesadas con controles nuevos recién constituidos.

En referencia a las bacterias es importante recalcar que la capacidad del equipo es equivalente a 400 campos de lectura en el microscopio, además el método de medición para sedimento utiliza un reactivo que tiñe todas las bacterias incluso las que no se ven en el microscopio, por tal razón, la concordancia de estos parámetros se pudo ver afectada. Por este motivo, la confirmación manual del sedimento se hace necesaria para corroborar las lecturas positivas que reporta el equipo. 
De acuerdo al fabricante hay diferentes condiciones en las muestras que pueden afectar el desempeño de lectura del equipo, especialmente en la sección FCM, como por ejemplo: muestras de alta densidad con piuria, muestras macroscópicas con hematuria, muestras con alta concentración de restos de mucosa, entre otras.(7) El análisis de orina de rutina consta de dos componentes principales: determinaciones fisicoquímicas (gravedad específica y mediciones de tiras reactivas) y un examen microscópico de sedimento de orina para evidencia de hematuria, piuria, moldes (cilindruria), cristaluria, etc. Sin embargo, el análisis microscópico es laborioso, lento, y necesita un alto nivel de experiencia para obtener interpretaciones. No obstante, la precisión del manual de la microscopía es baja debido a las variaciones en la preparación de la muestra y técnicas de conteo de partículas. A pesar de estas desventajas, el análisis microscópico todavía se usa en todo el mundo y sigue siendo el método de referencia para examinar las células y partículas en la orina. Varios sistemas automáticos de análisis de sedimentos de orina basado en tecnologías como la citometría de flujo y los sistemas de análisis basados en imágenes se usan actualmente en laboratorios clínicos. Estos sistemas automatizados ahorran tanto trabajo y tiempo, son más precisos, y permiten una mayor muestra del rendimiento que el análisis microscópico manual (8)

En un estudio realizado en Corea se comparó dos sistemas automatizados para orinas que emplean la citometría de flujo con el análisis microscópico manual. Dentro de los coeficientes de variación (CV), los materiales de control fueron 7.0-8.8\% y 1.7-5.7\%. Entre los coeficientes de variación corridos estuvieron $8.5-9.8 \%$ y $2.7-5.4 \%$. Ambos instrumentos mostraron una buena linealidad y una transferencia insignificante. Para los glóbulos rojos (RBC), glóbulos blancos (WBC) y células epiteliales (EPI), las tasas de concordancia total dentro del grado de diferencia entre los métodos automatizados y el manual fueron buenas (78.6 -86.0\%, 88.7-93.8\% y 81.3-90.7\%, respectivamente). La tasa de concordancia para los cilindros fue pobre (66.5-68.9\%). En comparación con la microscopía manual, los dos analizadores automáticos probados en este estudio mostraron rendimientos analíticos satisfactorios para glóbulos rojos, glóbulos blancos y células epiteliales. Sin embargo, para otras partículas de sedimento de orina, todavía se requiere la confirmación mediante microscopía visual.(8)

Wesarachkitti y colaboradores estudiaron 258 muestras en el año 2016. Evaluaron y compararon dos equipos automatizados. Se indicó que la correlación de las propiedades físicas / químicas de la orina entre los dos instrumentos fue excelente. El nivel de acuerdo para los glóbulos rojos en el equipo Cobas 6500 fue de: $R=0.94$ y en el UX-2000: $R=0.78$. Para los glóbulos blancos en el Cobas 6500 fue de: $\mathrm{R}=0.95$ y en el UX-2000: $\mathrm{R}=0.85$. El UX-2000 demostró una mayor sensibilidad para células redondas pequeñas, cilindros hialinos, cilindros patológicos y bacterias. El tiempo medio de respuesta fue de 1.5 minutos y 8.5 minutos para los Cobas 6500 y UX-2000, respectivamente. Se demostró que se reduce la carga de trabajo y aumenta el ahorro de tiempo. Sin embargo, se recomendó el examen manual por parte de los técnicos para las muestras patológicas.(9)

En el Centro Clínico de la Universidad de Sarajevo se reportó que se procesaron 368 muestras consecutivas de orina. Se analizaron mediante microscopía y analizadores automáticos. Ciertos elementos del sedimento de orina separados por microscopía manual se compararon con los analizados por el sistema Aution híbrido AU-4050; eritrocitos, leucocitos, cristales, cilindros y bacterias. La precisión del sistema se probó usando dos materiales de orina de 
control comercial. Se compararon todos los elementos del examen químico, tales como: glucosa, proteínas, bilirrubina, urobilinógeno, $\mathrm{pH}$, cetonas, nitritos y gravedad específica. Se concluyó que el análisis de orina automatizado empleado puede ahorrar trabajo y tiempo y es valioso en laboratorios que manejan un número importante de muestras. La sensibilidad y especificidad del equipo automatizado eliminaría la necesidad de microscopía manual para la mayoría de las muestras. El sistema químico de análisis de orina mostró un excelente concordancia.(10)

La automatización del área de uroanálisis es un requerimiento actual del laboratorio clínico que permite satisfacer la demanda creciente de los exámenes de orina. Permite además la estandarización y el control de cada una de las operaciones implicadas. De acuerdo con los resultados obtenidos en este estudio, señalamos que el analizador AU-4050 es una herramienta valiosa de apoyo al trabajo del laboratorista. No se considera que reemplace sus funciones, por lo tanto, la valoración clínica basada en los resultados que reporta el equipo debe correr a cargo del médico o laboratorista encargado, quien valorará además el estado clínico y otros resultados de estudio del paciente. Por otro lado, es importante que se cumplan con los mantenimientos preventivos del equipo en los tiempos establecidos, con el fin de garantizar el desempeño óptimo del mismo.

\section{Referencias Bibliografía}

1. Manrique-Abril FG, Rodríguez Díaz J, Ospina Díaz JM. Rendimiento diagnóstico del parcial de orina como predictor de infección urinaria en pacientes de Tunja, Colombia. CES Med. 2014;28(1):21-33.

2. Fernández DJ, Chiazza S Di. Análisis de orina: estandarización y control de calidad. Acta Bioquím Clín Latinoam. 2014;48(2):213-21.

3. Valle A. TJL DEL. El laboratorio clínico se acerca a los servicios mediante equipos Ela: Experiencia en Clínica Las Condes. Rev Médica Clínica Las Condes. 2015;26(6):802-7.

4. Coric J, Hasanefendic B, Zoljic B, Hasanbegovic L. Analytical evaluation of the aution hybrid AU-4050 automated urine analyzer. Med J Inst Res Dev Clin Cent Univ Sarajev. 2013;19(4):298-301.

5. Becker GJ, Garigali G, Fogazzi GB. Advances in urine microscopy. Am J Kidney Dis. 2016;67(6):954-64.

6. Suárez LCHPA. Manual de toma, conservación y transporte de muestras [Internet]. 2016. Available from: http://www.hpas.gob.ec/index.php/programas/aci

7. Arkray y Sysmex Corporation. Analizador de orina integrado totalmente automatizado AUTION HYBRID AU-4050 Instrucciones de uso. Japan; 2011. p. 7.35-7.47.

8. Lee W, Ha JS, Ryoo NH. Comparison of the Automated cobas u 701 Urine Microscopy and UF-1000i Flow Cytometry Systems and Manual Microscopy in the Examination of Urine Sediments. J Clin Lab Anal. 2016;30(5):663-71.

9. Wesarachkitti B, Khejonnit V, Pratumvinit B, Reesukumal K, Meepanya S, Pattanavin C, et al. Performance evaluation and comparison of the fully automated urinalysis analyzers UX-2000 and Cobas 6500. Lab Med. 2016;47(2):124-33.

10. Coric J, Hasanefendic B, Zoljic B, Hasanbegovic L. Analytical evaluation of the 
aution hybrid AU-4050 automated urine analyzer. Med J Inst Res Dev Clin Cent Univ Sarajev [Internet]. 2013;19(4):298-301. Available from: http://kcus.djikic.com/updf/Medicinski zurnal Vol 19 br 4 2013.pdf\#page $=33$

11. Lozano-triana CJ, Tract U, Link S, Imbiomed F. Examen general de orina : una prueba útil en niños Urinalysis : a useful test in children diagnosis. 2016;64(1):13747.

12. Fernández DJ, Chiazza S Di. Análisis de orina: estandarización y control de calidad. Acta Bioquím Clín Latinoam. 2014;48(2):213-21.

13. Valle A. TJL DEL. El laboratorio clínico se acerca a los servicios mediante equipos Ela: Experiencia en Clínica Las Condes. Rev Médica Clínica Las Condes. 2015;26(6):802-7.

14. Coric J, Hasanefendic B, Zoljic B, Hasanbegovic L. Analytical evaluation of the aution hybrid AU-4050 automated urine analyzer. Med J Inst Res Dev Clin Cent Univ Sarajev. 2013;19(4):298-301.

15. Becker GJ, Garigali G, Fogazzi GB. Advances in urine microscopy. Am J Kidney Dis. 2016;67(6):954-64.

16. Arkray y Sysmex Corporation. Analizador de orina integrado totalmente automatizado AUTION HYBRID AU-4050 Instrucciones de uso. Japan; 2011. p. 7.35-7.47.

17. Lee W, Ha JS, Ryoo NH. Comparison of the Automated cobas u 701 Urine Microscopy and UF-1000i Flow Cytometry Systems and Manual Microscopy in the Examination of Urine Sediments. J Clin Lab Anal. 2016;30(5):663-71.

18. Wesarachkitti B, Khejonnit V, Pratumvinit B, Reesukumal K, Meepanya S, Pattanavin $\mathrm{C}$, et al. Performance evaluation and comparison of the fully automated urinalysis analyzers UX-2000 and Cobas 6500. Lab Med. 2016;47(2):124-33.

19. Coric J, Hasanefendic B, Zoljic B, Hasanbegovic L. Analytical evaluation of the aution hybrid AU-4050 automated urine analyzer. Med J Inst Res Dev Clin Cent Univ Sarajev [Internet]. 2013;19(4):298-301. Available from: http://kcus.djikic.com/updf/Medicinski zurnal Vol 19 br 4 2013.pdf\#page=33

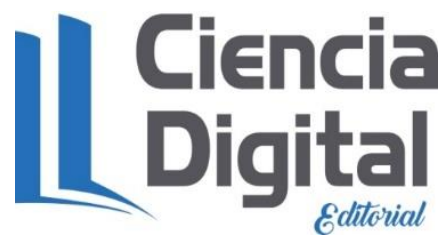


PARA CITAR EL ARTÍCULO INDEXADO.

Montalvo Torres, M., Peralta Mosquera, M., Robalino Montalvo, S., \& Ordoñez Revelo, M. (2019). Comparación del análisis de orina por el método manual y el automatizado. Ciencia Digital, 3(3.3), 177-186. https://doi.org/10.33262/cienciadigital.v3i3.3.791

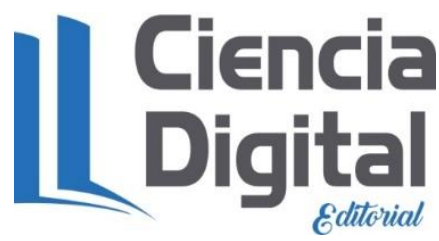

El artículo que se publica es de exclusiva responsabilidad de los autores y no necesariamente reflejan el pensamiento de la Revista Ciencia Digital.

El artículo queda en propiedad de la revista y, por tanto, su publicación parcial y/o total en otro medio tiene que ser autorizado por el director de la Revista Ciencia Digital.
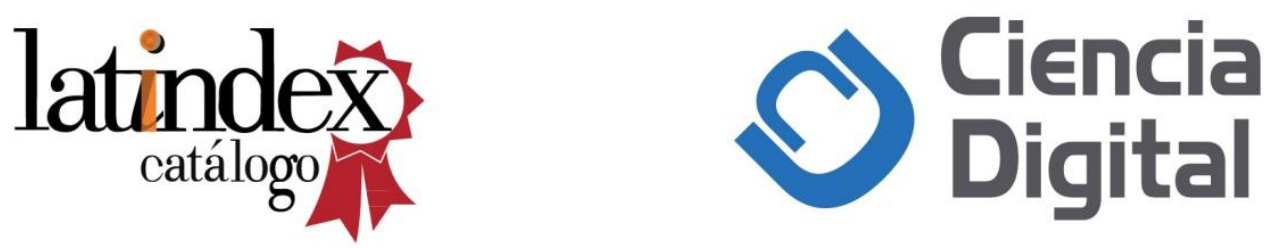